\title{
Modeling SARS-CoV-2 infection in vitro with a human intestine-on-chip device
}

Yaqiong Guo ${ }^{1,4} \uparrow$, Ronghua Luo $^{5,6,7} \dagger$, Yaqing Wang ${ }^{1,4} \dagger$, Pengwei Deng ${ }^{1,4} \dagger$, Min Zhang ${ }^{1,4}$, Peng Wang ${ }^{1}$, Xu Zhang ${ }^{1}$, Kangli Cui ${ }^{1,4}$, Tingting Tao ${ }^{1,4}$, Zhongyu $\mathrm{Li}^{1}$, Wenwen Chen ${ }^{1,4}$, Yongtang Zheng ${ }^{5,6,7^{*}}$, Jianhua Qin ${ }^{1,2,3,4, *}$

${ }^{1}$ CAS Key Laboratory of SSAC, Dalian Institute of Chemical Physics, Chinese Academy of Sciences, Dalian, China.

${ }^{2}$ Institute For Stem Cell and Regeneration, Chinese Academy of Sciences, Beijing, China.

${ }^{3}$ CAS Center for Excellence in Brain Science and Intelligence Technology, Chinese Academy of Sciences, Shanghai, China.

${ }^{4}$ University of Chinese Academy of Sciences, Beijing, China.

${ }^{5}$ Kunming National High-level Bio-safety Research Center for Non-human Primates, Center for Biosafety Mega-Science, Kunming Institute of Zoology, Chinese Academy of Sciences, Kunming, 650107, China

${ }^{6}$ Key Laboratory of Animal Models and Human Disease Mechanisms of Chinese Academy of Sciences and Yunnan Province, Kunming Institute of Zoology, Chinese Academy of Sciences, Kunming, Yunnan 650223, China

${ }^{7}$ KIZ-CUHK Joint Laboratory of Bioresources and Molecular Research in Common Diseases, Kunming Institute of Zoology, Chinese Academy of Sciences, Kunming, Yunnan 650223, China

*Correspondence:

Jianhua Qin, Division of Biotechnology, Dalian Institute of Chemical Physics, Chinese Academy of Sciences, China. E-mail: jhqin@dicp.ac.cn.

Yongtang Zheng, Kunming Institute of Zoology, 32 Jiaochang Donglu Kunming, Yunnan, E-mail: zhengyt@mail.kiz.ac.cn.

$\dagger$ These authors contribute equally to this work.

The authors have no conflicts of interest to declare. 


\begin{abstract}
Coronavirus disease 2019 (COVID-19) caused by severe acute respiratory syndrome coronavirus (SARS-CoV-2) has given rise to a global pandemic. The gastrointestinal symptoms of some COVID-19 patients are underestimated. There is an urgent need to develop physiologically relevant model that can accurately reflect human response to viral infection. Here, we report the creation of a biomimetic human intestine infection model on a chip system that allows to recapitulate the intestinal injury and immune response induced by SARS-CoV-2, for the first time. The microengineered intestineon-chip device contains human intestinal epithelium (co-cultured human intestinal epithelial Caco-2 cells and mucin secreting HT-29 cells) lined in upper channel and vascular endothelium (human umbilical vein endothelial cells, HUVECs) in a parallel lower channel under fluidic flow condition, sandwiched by a porous PDMS membrane coated with extracellular matrix (ECM). At day 3 post-infection of SARS$\mathrm{CoV}-2$, the intestine epithelium showed high susceptibility to viral infection and obvious morphological changes with destruction of intestinal villus, dispersed distribution of mucus secreting cells and reduced expression of tight junction (Ecadherin), indicating the destruction of mucous layer and the integrity of intestinal barrier caused by virus. Moreover, the endothelium exhibited abnormal cell morphology with disrupted expression of adherent junction protein (VE-cadherin). Transcriptional analysis revealed the abnormal RNA and protein metabolism, as well as activated immune responses in both epithelial and endothelial cells after viral infection (e.g., up-regulated cytokine genes, TNF signaling and NF-kappa B signaling-related genes). This bioengineered in vitro model system can mirror the human relevant pathophysiology and response to viral infection at the organ level, which is not possible in existing in vitro culture systems. It may provide a promising tool to accelerate our understanding of COVID-19 and devising novel therapies.
\end{abstract}

\title{
INTRODUCTION
}

COVID-19 caused by SARS-CoV-2 has become a global epidemic. As of late August 2020, it has caused 22 million infections and more than 790,000 deaths. SARS-CoV-2 infection is characterized by a process ranging from mild disease to severe systemic symptoms of multiple organs, most notably the lung, gastronintestinal tract, etc., and finally multi-organ failure (1). Respiratory symptoms dominate the clinical manifestations of COVID-19, yet obvious gastrointestinal symptoms were observed in $20 \%$ to $50 \%$ of patients, including abdominal pain, diarrhea, hematochezia and even intestinal perforation (2-5). In particular, these symptoms sometimes appear before the onset of respiratory symptoms (6). In addition, biopsy samples showed a large number of interstitial edema plasma cells and lymphocytes infiltrated into lamina propria of the stomach, duodenum and rectum (7). It has been reported that 
SARS-CoV-2 can cause acute hemorrhagic colitis, providing the evidence of the gastrointestinal tract implicating in the transmission of SARS-CoV-2 infection (8). Moreover, the viral RNA was identified in stool samples of COVID-19 patients and typical coronavirus virions in rectal tissue were observed under electron microscopy, implying that SARS-CoV-2 could potentially be transmitted via the fecal-oral route (9-11). These clinical evidences suggest that intestine represents another high-risk organ for SARS-CoV-2 infection besides the lungs, but the pathogenesis of intestinal infection underlying COVID-19 is elusive.

In vivo, human intestine contains complex multicellular components and hostpathogen interactions in a physiological flow microenvironment with mechanical cues. Currently, SARS-CoV-2 infection in intestine is studied based on monolayer cultures of intestinal epithelial cells $(12,13)$ and human organoids $(14,15)$. However, these in vitro models still have their limitations. The monolayer cell culture is oversimplified and cannot recapitulate the multiple cellular component, complex structure and functions of native intestine. Moreover, they lack cell-cell/matrix interactions and tissue-specific dynamic microenvironment that exist in vivo. Recently, intestinal organoids have provided a new in vitro 3D model for SARS-CoV2 infection by providing multiple cell types and supporting viral replication in the gut enterocytes (14). But these organoids are still limited by the lack of typical characteristics of intestinal barrier, ECM, immune cells and physiological flow, which are key features of the intestinal microenvironment. As such, it is highly desirable to develop alternative in vitro models to better reflect the pathophysiology of human organs to SARS-CoV-2 infection.

Organ-on-a-chip technology has evolved to provide the possibility to reproduce the complex structures and physiological functions units of human organs in engineered microfluidic culture device (16-18). It has been utilized to represent the organ-level physiology and pathology, and applied in various biomedical applications, including organ engineering, disease studies and drug testing (19-23). In this work, we established a perfusable intestine infection model on chip system that simulate the human-relevant intestine response to SARS-CoV-2 infection at organ level. The microengineered intestine chip device consists of human intestine epithelium layer and vascular endothelium layer separated by an ECM coated porous PDMS membrane, in which Caco-2 cells and HT-29 cells are co-cultured in the upper channel, while HUVEC cells and circulating immune cells are lined in the lower channel under fluid flow. Using this system, we examined the infection and replication of SARS-CoV-2 in epithelial cells. We then systematically analyzed the changes of intestinal epithelium and endothelium induced by virus infection using confocal imaging. We also characterized the pathological changes and immune responses of intestinal barrier after viral infection via RNA-sequencing analysis. This 
human disease model on chip offers a novel strategy and platform for organ-level COVID-19 research and potential therapeutics.

\section{RESULTS}

\section{Characterization of human intestine-on-chip device}

In vivo, human intestinal epithelium is a cell monolayer that constitutes the intestinal barrier, which allows the mucus secretion, absorption of nutrients and prevents the invasion of pathogenic antigen or toxins in a dynamic microenvironment. In order to create an in vitro human model of intestine infection induced by SARS-CoV-2, we initially designed and constructed an intestine chip in a perfusable multilayer microfluidic culture device. Briefly, the device consists of two parallel channels separated by a thin and porous PDMS membrane coated with ECM to form biointerface and facilitate the nutrient exchange between upper and lower cell layers.

In this work, the intestine epithelial cells Caco-2 and intestinal mucin secreting cells HT-29 were co-cultured to build intestinal epithelium in the upper channel under fluid flow $(200 \mu \mathrm{L} / \mathrm{h})$ to simulate the intraluminal fluid flow in intestine. The HUVEC cells were cultured in the lower channel to form vascular endothelium under fluid flow $(50 \mu \mathrm{L} / \mathrm{h})$. After 3 days of co-culture, the intestine epithelial and endothelial cells were confluent into monolayers under fluid flow, thus forming an intestine epithelium-endothelium tissue barrier. The integrity of the epithelial barrier was identified by assessing the expression of tight junction (ZO-1) and adherent junction (E-cadherin) proteins. The immunostaining analysis showed the positive expression of ZO-1 and E-cadherin in epithelium (Fig. 2A). In particular, the epithelial cells formed villus-like structure lined by a highly polarized columnar epithelium identified by DIC imaging, which was similar to in vivo intestinal villi (Fig. 2C). Moreover, the integrity of endothelium was examined by ZO-1 and VE-cadherin immunostaining, showing the regular and intact distribution of junction proteins (Fig. 2B). As the intestinal mucus layer is the crucial interface between the host and microbes, which plays a vital role in preventing microbial invasion (24). We examined the mucus production in intestinal epithelium by immunostaining with mucin specific marker (MUC). As shown in Fig. 2D, it exhibited a scattered distribution of MUC positive cells in the intestinal epithelium, suggesting the secretory function of mucus. It appears that the established intestine chip system exhibited intestinal villus-like structure, the integrity of tissue barrier and ability of mucus secretion under fluidic flow, representing the key features of human intestinal barrier in a physiologically relevant manner.

SARS-CoV-2 infection in the intestine-on-chip 
Prior to SARS-CoV-2 infection on the intestine chip, we first detected the expression level of ACE2 and TMPRSS2 in three cell lines by western blot, including Caco-2, HT-29 and HUVEC cells. It is recognized that SARS-CoV-2 uses ACE2 as a receptor for cellular entry and TMPRSS 2 protease for viral Spike protein priming $(25,26)$. The intestinal enterocytes have a higher expression of ACE2 compared to alveolar epithelial type II cells (27-29). Among the tested three cell lines in this work, ACE2 and TMPRSS2 were expressed at the highest level in Caco-2 cells (Fig. S1A), which implied that Caco- 2 cells may be more susceptible to SARS-CoV-2 infection. Caco-2 cells were then inoculated with different multiplicity of virus (MOI=0.04, 0.4 and 2) to identify the virus susceptibility. Three days after infection, immunostaining data showed more than $80 \%$ Spike protein-positive cells were observed in Caco-2 cells at MOI of 2 (Fig. S1B).

To examine the SARS-CoV-2 infection on the intestine chip, the virus was inoculated into the intestinal channel at $\mathrm{MOI}=1$ for $24 \mathrm{~h}$ after co-culture of intestinal epithelium and endothelium for 3 days. The virus infection in the intestinal epithelium layer and endothelium layer were then examined by immunostaining analysis. The data showed that Spike positive cells were predominantly detected on the intestinal layer, indicating that the intestinal epithelial cells are more susceptible to SARS-CoV2 infection than endothelial cells (Fig. 3).

In vivo, the development and barrier maintenance of intestine depends heavily on the proper function of adherent junctions, we further examined the adherent junctions of intestinal epithelium and endothelium, respectively. Confocal micrographs of intestinal epithelium showed that on day 3 after viral infection, the adherent junction identified by E-cadherin was severely destroyed, accompanied by the damage of intestinal villus-like structures (Fig. 3A). Moreover, the mucous secreting cells in intestinal epithelium were examined by immunostaining with MUC2. The results showed that the clonal distribution $\mathrm{MUC}^{+}$cells became dispersed after viral infection (Fig. 4A). This suggests that the disturbed intestinal mucus layer was caused by viral infection, which may lead to virus encroachment and infection. It is noted that, in the vascular side, the adherent junctions between endothelial cells were severely disrupted by VE-cadherin immunostaining (Fig. 4B). Quantitative analysis showed that the density and size of endothelial cells were significantly decreased (Fig. 4C and 4D). It reveals the injury of vascular endothelial cells following viral infection, while there were no obvious Spike positive cells on the vascular side. The presence of endothelial injury might partially explain the pathogenesis of COVID-19 associated coagulopathy or vascular thrombosis (30).

\section{Transcriptional analysis of host cells to SARS-CoV-2 infection}

To gain a comprehensive overview of transcriptional responses to SARS-CoV-2 infection, we performed RNA-sequencing analysis of intestinal epithelial cells and 
endothelial cells in human intestine model. Briefly, 3 days after infection, intestinal epithelial cells and endothelial cells were collected separately and analyzed by RNAsequencing. Volcano plots showed that SARS-CoV-2 infection induced dramatic transcriptome modulations in both intestinal epithelial cells and endothelial cells (Fig. 5A). In order to identify differentially expressed genes (DEGs), the critical value and $\mathrm{P}$ value of the abundance fold change were set to 2.0 and 0.05 , respectively. Among the DEGs, 9684 genes (4022 down-regulated genes and 5662 up-regulated genes) were significantly modulated in intestinal epithelial cells, while 6713 genes (2569 down-regulated genes and 4144 up-regulated genes) were significantly modulated in endothelial cells following viral infection. This result suggested that SARS-CoV-2 infection had a greater effect on intestinal epithelial cells than endothelial cells, possibly due to higher viral load in intestinal epithelium. By combining the two data sets, we found that intestinal epithelial cells and endothelial cells shared 1791 overlapping up-regulated DEGs (37.3\% of total up-regulated DEGs) and 3317 overlapping down-regulated DEGs (51.5\% of total down-regulated DEGs) (Fig. 5B).

To investigate the host biological pathways modified by SARS-CoV-2 infection, KEGG pathway enrichments analysis comparing mock-infected versus SARS-CoV-2 infected intestine chips was performed (Fig. 5C and 5D). The results revealed that the viral infection resulted in the abnormal pathway networks in both cell types, including RNA metabolism pathways (e.g., spliceosome, RNA transport, RNA degradation, mRNA surveillance pathway) and protein metabolism pathways (e.g., ubiquitin mediated proteolysis, protein export). Given the vital roles of RNA and protein metabolism in maintaining the normal physiological functions of cells, we reasoned that SARS-CoV-2 may seriously affect the host cells by disrupting these critical pathways. In addition, we found that some immune responses related pathways, such as TNF signaling pathway and NF-kappa B signaling pathway, were particularly enriched in genes that are significantly up-regulated in endothelial cells, which indicated that the intestinal endothelial cells play critical roles in mediating intestinal immune responses.

\section{Immune response of intestinal model on chip to SARS-CoV-2 infection}

According to the above transcriptional analysis, the KEGG pathway enrichment analysis indicated some immune response-related signaling pathways were activated following SARS-CoV-2 infection. We then tried to identify the up-regulated cytokine genes that mediated the subsequent inflammatory responses underlying SARS-CoV2 -induced intestinal infection. The heat map showed that SARS-CoV-2 infection elicited an extensive cytokines induction in both intestinal epithelium and endothelium, including TNF, interleukins, chemokines and colony-stimulating factors in both intestinal epithelial cells and endothelial cells (Fig. 6A and 6B). Notably, in this infected intestinal model, some cytokines including TNF, IL-6, CXCL10, CCL5 
and CSF3 were significantly up-regulated, which is similar to the clinical manifestations of patients with severe COVID-19 $(31,32)$. These results may partially explain the presence of infiltrated immune cells and inflammatory response in the intestine of patients with severe COVID-19.

Then, we further detected the expression of pro-inflammatory cytokines and chemokines-related genes in the intestinal epithelial cells by qRT-PCR. The expression of CCL5, CXCL1, CXCL10 and CXCL11 were significantly up-regulated in the SARS-CoV-2-infected epithelial cells (Fig. 6C). The data indicated that chemokine genes could be induced by SARS-CoV-2, which may play a crucial role in the recruitment of immune cells and modulation of immune response to virus infection. It has been previously reported that chemokines CCL5 can recruit T cells, dendritic cells, eosinophils, NK cells, mast cells, and basophils (33), CXCL1 recruits neutrophils (34), CXCL10 recruits activated Th1 lymphocytes (35), and CXCL11 recruits interleukin-activated T-cells (36) to sites of inflammation. This might provide the clues to further identify which immune cells are involved in the intestinal inflammatory responses in the progression of COVID-19.

\section{DISCUSSION}

In this study, we created a biomimetic human disease model of intestinal infection on chip by SARS-CoV-2, which could mirror the pathophysiological features and immune response associated with gastrointestinal symptoms in COVID-19. This in vitro model can reflect the damage of the intestinal epithelial barrier, including the destruction of intestinal villi, the disruption of barrier integrity, mucosal secretion disorder and activated immune responses after SARS-CoV-2 infection, which may help us to understand the pathological processes involved in COVID-19. Moreover, despite the low susceptibility to virus infection, the vascular endothelium displayed significant morphological damage induced by SARS-CoV-2, revealing the possible complex cross-talk between intestine epithelial cells and endothelial cells in mediating tissue barrier injury and COVID-19 progression.

In vivo, human intestine comprises the largest component of human immune system with large populations of scattered innate and adaptive effector cells, because it is constantly exposed to foreign antigens and other environmental agents. The protective mucus gel secreted by goblet cells is distributed on the gastrointestinal epithelium, which provides chemical and physical defenses for the intestinal barrier and plays a crucial role in intestinal homeostasis. In this intestine chip, the Caco- 2 cells and mucin secreting HT-29 cells were co-cultured under continuous perfusion to form intestinal epithelium, which can recapitulate the intestinal barrier in a highly physiological relevant manner. In particular, we found that the secretion of intestinal 
mucin has undergone unrecognized changes, from agglomerated distribution to scattered distribution after viral infection. It is speculated that the disturbed mucus layer may be associated with further invasion and infection of virus. These results may also explain the increased intestinal permeability and further cause symptoms, such as diarrhea ${ }^{6}$ and hemorrhagic colitis (37) in COVID-19 patients.

Transcriptome analysis demonstrated significantly altered vital biological processes in both intestinal epithelium and endothelium following SARS-CoV-2 infection, including RNA and protein metabolism pathways, cell cycle regulation and oxidative phosphorylation. Specifically, we identified many up-regulated cytokine and chemokine-related genes in the intestinal epithelial cells, similar to the clinical manifestations of COVID-19. Because chemokine can act as chemoattractant to recruit immune cells to the infected sites, these altered chemokines may contribute to the immune cells mediated inflammatory responses in the intestine. In addition, we found that the intestinal epithelium is more susceptible to SARS-CoV-2 infection than endothelium on chip, which is consistent with previous studies (12). As such, we assume that the vascular endothelial injury may be mediated by the inflammatory factors or paracrine signals released by virus infected intestinal epithelial cells.

One potential limitation of this work is the use of immortalized intestinal epithelial cell lines (e.g., Caco-2) originally isolated from human colon tumors. However, Caco2 cells have been widely used to recapitulate many physiological and pathological functions of human intestine in vivo $(38,39)$. The intestinal epithelium differentiated from primary intestinal stem cells may be selected for further studies in later time.

Overall, in this work, we provide the proof-of-concept to build an intestine-on-chip infection model at organ-level that permits to closely mirror the intestinal pathophysiology and human response to SARS-CoV-2 infection, which is impossible to achieve by existing in vitro culture models. It can not only supply a unique, rapid and low-cost in vitro platform for viral infection, but also provide a complement to animal models to study disease progress, virus transmission and host-virus interactions in a more realistic manner, thereby accelerating the COVID-19 research and development of novel therapeutics. 


\section{MATERIALS AND METHODS}

\section{Device fabrication}

The human intestine model consisted of the upper and lower layers fabricated by using conventional soft lithography procedures. Polydimethylsiloxane (PDMS) prepolymer was prepared by mixing 10:1 (wt/wt) PDMS base to curing agent (184 Silicone Elastomer, Dow Corning Corp) and casted on molds to produce molded device with channels by thermal curing at $80{ }^{\circ} \mathrm{C}$ for $45 \mathrm{~min}$. The channels were 1.5 $\mathrm{mm}$ wide $\times 0.25 \mathrm{~mm}$ high and the length of overlapping channels was $8 \mathrm{~mm}$. The two channels were separated by a thin $(\sim 20 \mu \mathrm{m})$ through-hole PDMS membrane (pore size: $5 \mu \mathrm{m}$ ) to construct tissue-tissue interfaces. The porous PDMS membranes were fabricated based on the glass templates and spin-coating method modified from the previous protocol. The membrane was sandwiched in the intestine-on-chip device by oxygen plasma bonding. Finally, the devices were exposed to ultraviolet light for disinfection and sterilization. Prior to cell seeding, both sides of the porous membrane were coated with rat tail collagen type I ( $200 \mu \mathrm{g} / \mathrm{mL}$, Corning) and incubated at $37{ }^{\circ} \mathrm{C}$ for $48 \mathrm{~h}$.

\section{Cell culture}

Human colon adenocarcinoma cells (Caco-2) were cultured in high-glucose Dulbecco's Modified Eagle's Medium (DMEM, Gibco) supplemented with 10\% Fetal Bovine Serum (FBS, Gibco). Human colorectal adenocarcinoma grade II cells HT29 were purchased from Procell Life Science \& Technology Co., Ltd (Procell, CL-0118) and were maintained in growth medium (Procell, CM-0118) supplemented with $10 \%$ FBS. Human umbilical vein endothelial cells (HUVEC) were isolated from human umbilical cord and cultured in Endothelial Cell Medium purchased from ScienCell Research Laboratories, inc. Human peripheral blood mononuclear cells were isolated from fresh human blood using Ficoll (Stem cell technologies) density centrifugation. Isolated PBMCs were resuspended in RPMI 1640 medium containing 10\% FBS and $50 \mathrm{IU} / \mathrm{mL}$ IL-2 and used for adhesion assays on chip. All cells were cultured at $37{ }^{\circ} \mathrm{C}$ in a humidified atmosphere of $5 \% \mathrm{CO} 2$.To create the intestine model, HUVEC cells ( $1 \times 10^{5}$ cells) were initially seeded on the bottom side of the collagen-coated porous PDMS membrane and allowed to attach on the membrane surface under static conditions for two hours. Subsequently, cells were washed with fresh medium to exclude/remove unattached ones. Then, Caco- 2 cells $\left(\sim 1 \times 10^{5}\right.$ cells $)$ and HT29 cells $\left(\sim 1 \times 10^{4}\right.$ cells) were mixed and seeded into the upper channel under static cultures. After cell attachment, cells were grown to confluence for 3 days and the chips were maintained in an incubator with $5 \% \mathrm{CO} 2$ at $37^{\circ} \mathrm{C}$.

\section{Virus}


A clinical isolate SARS-COV-2 strain 107 was obtained from Guangdong Provincial Center for Disease Control and Prevention, China, and propagated in Vero E6 cells. The virus titers were (infectious titers of virus) were determined by a TCID50 assay on Vero cells. All work involving live SARS-CoV-2 was performed in the Chinese Center for Disease Control and Prevention-approved BSL-3 laboratory of the Kunming Institute of Zoology, Chinese Academy of Science.

\section{SARS-CoV-2 infection}

Caco-2 cells were seeded in 24 -well plates $\left(2 \times 10^{5}\right.$ cells per well) in high-glucose Dulbecco's Modified Eagle's Medium containing 10\% FBS. After seeding for 24h, cells were infected with SARS-CoV-2 at an indicated multiplicity of virus (MOI=0.04, 0.4 and 2). After one hour, cells were washed three times with PBS and kept in fresh medium for 3 days. At day 3 post-infection, cells were washed with PBS and then fixed with $4 \%$ paraformaldehyde (PFA) before analysis.

For SARS-CoV-2 infection in the human intestine model, the apical channel of chip device was infused with $30 \mu \mathrm{L}$ of high-glucose DMEM medium containing the indicated multiplicity of virus $(\mathrm{MOI}=1)$. After one hour of infection, cells were washed three times with PBS and kept in fresh medium. At day 3 post-infection, the epithelial cells and endothelial cells cultured on chip were fixed for immunofluorescence analysis or lysed for RNA- sequencing analysis, respectively.

\section{Immunostaining}

Caco-2 cells cultured on well plate were washed with PBS and fixed with 4\% PFA at $4^{\circ} \mathrm{C}$ overnight. Cells were then permeabilized with $0.2 \%$ Triton X-100 in PBS (PBST buffer) for $5 \mathrm{~min}$ and blocked with PBST buffer containing 5\% normal goat serum for 30 minutes at room temperature. Antibodies were diluted with PBST buffer. Cells were stained with corresponding primary antibodies at $4^{\circ} \mathrm{C}$ overnight and with secondary antibodies (supplementary Table S1) at room temperature for 1hour. After staining with secondary antibodies, cell nuclei were counterstained with DAPI. For immunofluorescent imaging of intestine model, cells were washed with PBS through the upper and bottom channels and fixed with 4\% PFA. The fixed tissues were subjected to immunofluorescence staining by the same procedure as described above. All images were acquired using a confocal fluorescent microscope system (FV-1000, Olympus). Image processing was done using ImageJ (NIH).

\section{RNA extraction, library preparation and sequencing}

Intestinal epithelial cells and endothelial cells were collected separately from the chips, and total RNAs were extracted from samples using Trizol (Invitrogen). DNA digestion was carried out after RNA extraction by DNaseI. RNA quality was determined by examining A260/A280 with NanodropTM OneCspectrophotometer (Thermo Fisher Scientific Inc). RNA Integrity was confirmed by $1.5 \%$ agarose gel 
electrophoresis. Qualified RNAs were finally quantified by Qubit3.0 with QubitTM RNA Broad Range Assay kit (Life Technologies). 500 ng total RNAs were used for stranded RNA sequencing library preparation using KC-DigitalTM Stranded mRNA Library Prep Kit for Illumina ${ }^{\circledR}$ (Catalog NO. DR08502, Wuhan SeqHealth Co., Ltd. China) following the manufacturer's instruction. The kit eliminates duplication bias in PCR and sequencing steps, by using unique molecular identifier (UMI) of 8 random bases to label the pre-amplified cDNA molecules. The library products corresponding to 200-500 bps were enriched, quantified and finally sequenced on Hiseq X 10 sequencer (Illumina).

\section{RNA-sequencing analysis}

Raw sequencing data was first filtered by Trimmomatic (version 0.36), low-quality reads were discarded and the reads contaminated with adaptor sequences were trimmed. Clean Reads were further treated with in-house scripts to eliminate duplication bias introduced in library preparation and sequencing. In brief, clean reads were first clustered according to the UMI sequences, in which reads with the same UMI sequence were grouped into the same cluster, resulting in 65,536 clusters. Reads in the same cluster were compared to each other by pairwise alignment, and then reads with sequence identity over $95 \%$ were extracted to a new sub-cluster. After all sub-clusters were generated, multiple sequence alignment was performed to get one consensus sequence for each sub-cluster. After these steps, any errors and biases introduced by PCR amplification or sequencing were eliminated.

The de-duplicated consensus sequences were used for standard RNA-sequencing analysis. They were mapped to the reference genome of Homo sapiens from Ensembl database (ftp://ftp.ensembl.org/pub/release-87/fasta/homo_sapiens/dna/) using STAR software (version 2.5.3a) with default parameters. Reads mapped to the exon regions of each gene were counted by featureCounts (Subread-1.5.1; Bioconductor) and then RPKMs were calculated. Genes differentially expressed between groups were identified using the edgeR package (version 3.12.1). An FDR corrected p-value cutoff of 0.05 and Fold-change cutoff of 2 were used to judge the statistical significance of gene expression differences. Gene ontology (GO) analysis and Kyoto encyclopedia of genes and genomes (KEGG) enrichment analysis for differentially expressed genes were both implemented by KOBAS software (version: 2.1.1) with a corrected P-value cutoff of 0.05 to judge statistically significant enrichment.

\section{Statistical analyses}

Data were collected in Excel (Microsoft). Differences between two groups were analyzed using a Student's t-test. Multiple group comparisons were performed using a one-way analysis of variance (ANOVA) followed by post-hoc tests. The bar graphs 
with error bars represent mean \pm standard deviation (SD). Significance is indicated by asterisks: *, $\mathrm{p}<0.05 ; * *, \mathrm{p}<0.01 ; * * *, \mathrm{p}<0.001$.

\section{Data availability}

All relevant data are available in the manuscript or supporting information. All of the RNA-sequencing raw data have been deposited on SRA under the accession number PRJNA658711.

\section{Supporting information}

Supporting information is available from the Wiley Online Library or from the author.

\section{Acknowledgments}

This research was supported by the Strategic Priority Research Program of the Chinese Academy of Sciences, Grant (Nos. XDB29050301, XDA16020900, XDB32030200), National Key R\&D Program of China (No. 2017YFB0405404), The National key Research and Development Program of China (2020YFC0842000), National Science and Technology Major Project (No. 2018ZX09201017-001-001), National Nature Science Foundation of China (Nos. 31671038, 31971373, 81703470, 81803492), China Postdoctoral Science Foundation (No. 2019M660065), Innovation Program of Science and Research from the DICP, CAS (DICP I201934). We thank Prof. Yonggang Yao (Kunming Institute of Zoology, CAS) for his strong support on this work.

\section{Conflict of interest}

The authors declare no conflict of interest.

\section{References}

1. W. J. Wiersinga, A. Rhodes, A. C. Cheng, S. J. Peacock, H. C. Prescott, Pathophysiology, transmission, diagnosis, and treatment of coronavirus disease 2019 (COVID-19): a review. Jama, (2020).

2. L. Lin, X. Jiang, Z. Zhang, S. Huang, Z. Zhang, Z. Fang, Z. Gu, L. Gao, H. Shi, L. Mai, Gastrointestinal symptoms of 95 cases with SARS-CoV-2 infection. Gut 69, 997-1001 (2020).

3. F. D'Amico, D. C. Baumgart, S. Danese, L. Peyrin-Biroulet, Diarrhea during COVID-19 infection: pathogenesis, epidemiology, prevention and management. Clinical Gastroenterology and Hepatology, (2020).

4. S. C. Ng, H. Tilg, COVID-19 and the gastrointestinal tract: more than meets the eye. Gut 69, 973-974 (2020).

5. Y. Wan, J. Li, L. Shen, Y. Zou, L. Hou, L. Zhu, H. S. Faden, Z. Tang, M. Shi, N. Jiao, Enteric involvement in hospitalised patients with COVID-19 outside Wuhan. The lancet Gastroenterology \& hepatology 5, 534-535 (2020). 
6. Y. Song, P. Liu, X. Shi, Y. Chu, J. Zhang, J. Xia, X. Gao, T. Qu, M. Wang, SARS-CoV-2 induced diarrhoea as onset symptom in patient with COVID-19. Gut 69, 1143-1144 (2020).

7. F. Xiao, M. Tang, X. Zheng, Y. Liu, X. Li, H. Shan, Evidence for gastrointestinal infection of SARS-CoV-2. Gastroenterology 158, 1831-1833. e1833 (2020).

8. Q. Ye, B. Wang, T. Zhang, J. Xu, S. Shang, The mechanism and treatment of gastrointestinal symptoms in patients with COVID-19. American journal of physiology. Gastrointestinal and liver physiology, (2020).

9. K. S. Cheung, I. F. Hung, P. P. Chan, K. Lung, E. Tso, R. Liu, Y. Ng, M. Y. Chu, T. W. Chung, A. R. Tam, Gastrointestinal manifestations of SARS-CoV2 infection and virus load in fecal samples from the Hong Kong cohort and systematic review and meta-analysis. Gastroenterology, (2020).

10. J. Hindson, COVID-19: faecal-oral transmission? Nature Reviews Gastroenterology \& Hepatology 17, 259-259 (2020).

11. Q. Qian, L. Fan, W. Liu, J. Li, J. Yue, M. Wang, X. Ke, Y. Yin, Q. Chen, C. Jiang, Direct evidence of active SARS-CoV-2 replication in the intestine. Clinical infectious diseases : an official publication of the Infectious Diseases Society of America, (2020).

12. M. L. Stanifer, C. Kee, M. Cortese, C. M. Zumaran, S. Triana, M. Mukenhirn, H.-G. Kraeusslich, T. Alexandrov, R. Bartenschlager, S. Boulant, Critical role of type III interferon in controlling SARS-CoV-2 infection in human intestinal epithelial cells. Cell reports 32, (2020).

13. R. Zang, M. F. Gomez Castro, B. T. McCune, Q. Zeng, P. W. Rothlauf, N. M. Sonnek, Z. Liu, K. F. Brulois, X. Wang, H. B. Greenberg, M. S. Diamond, M. A. Ciorba, S. P. J. Whelan, S. Ding, TMPRSS2 and TMPRSS4 promote SARS-CoV-2 infection of human small intestinal enterocytes. Science immunology 5, (2020).

14. M. M. Lamers, J. Beumer, J. van der Vaart, K. Knoops, J. Puschhof, T. I. Breugem, R. B. G. Ravelli, J. Paul van Schayck, A. Z. Mykytyn, H. Q. Duimel, E. van Donselaar, S. Riesebosch, H. J. H. Kuijpers, D. Schipper, W. J. van de Wetering, M. de Graaf, M. Koopmans, E. Cuppen, P. J. Peters, B. L. Haagmans, H. Clevers, SARS-CoV-2 productively infects human gut enterocytes. Science (New York, N.Y.) 369, 50-54 (2020).

15. J. Zhou, C. Li, X. Liu, M. C. Chiu, X. Zhao, D. Wang, Y. Wei, A. Lee, A. J. Zhang, H. Chu, Infection of bat and human intestinal organoids by SARSCoV-2. Nature medicine, 1-7 (2020).

16. S. N. Bhatia, D. E. Ingber, Microfluidic organs-on-chips. Nature Biotechnology 32, 760-772 (2014).

17. A. Schepers, C. Li, A. Chhabra, B. T. Seney, S. Bhatia, Engineering a perfusable 3D human liver platform from iPS cells. Lab Chip 16, 2644-2653 (2016).

18. B. Zhang, A. Korolj, B. F. L. Lai, M. Radisic, Advances in organ-on-a-chip engineering. Nature Reviews Materials 3, 257-278 (2018).

19. D. Huh, B. D. Matthews, A. Mammoto, M. Montoya-Zavala, H. Y. Hsin, D. E. Ingber, Reconstituting organ-level lung functions on a chip. Science 328, 1662-1668 (2010).

20. L. Wang, T. Tao, W. Su, H. Yu, Y. Yu, J. Qin, A disease model of diabetic nephropathy in a glomerulus-on-a-chip microdevice. Lab on a Chip 17, 17491760 (2017). 
21. T. Tao, Y. Wang, W. Chen, Z. Li, W. Su, Y. Guo, P. Deng, J. Qin, Engineering human islet organoids from iPSCs using an organ-on-chip platform. Lab on a Chip, (2019).

22. R. Novak, M. Ingram, S. Marquez, D. Das, A. Delahanty, A. Herland, B. M. Maoz, S. S. Jeanty, M. R. Somayaji, M. Burt, Robotic fluidic coupling and interrogation of multiple vascularized organ chips. Nature Biomedical Engineering, 1-14 (2020).

23. Y. Guo, Z. Li, W. Su, L. Wang, Y. Zhu, J. Qin, A Biomimetic Human Gut-ona-Chip for Modeling Drug Metabolism in Intestine. Artificial Organs, (2018).

24. E. M. Brown, M. Sadarangani, B. B. Finlay, The role of the immune system in governing host-microbe interactions in the intestine. Nature Immunology 14, 660-667 (2013).

25. M. Hoffmann, H. Kleine-Weber, S. Schroeder, N. Krüger, T. Herrler, S. Erichsen, T. S. Schiergens, G. Herrler, N.-H. Wu, A. Nitsche, SARS-CoV-2 cell entry depends on ACE2 and TMPRSS2 and is blocked by a clinically proven protease inhibitor. Cell, (2020).

26. P. Zhou, X.-L. Yang, X.-G. Wang, B. Hu, L. Zhang, W. Zhang, H.-R. Si, Y. Zhu, B. Li, C.-L. Huang, A pneumonia outbreak associated with a new coronavirus of probable bat origin. nature 579, 270-273 (2020).

27. S. B. Chadchan, V. K. Maurya, P. Popli, R. Kommagani, The SARS-CoV-2 receptor, Angiotensin converting enzyme 2 (ACE2) is required for human endometrial stromal cell decidualization. bioRxiv : the preprint server for biology, (2020).

28. R. Yan, Y. Zhang, Y. Li, L. Xia, Y. Guo, Q. Zhou, Structural basis for the recognition of SARS-CoV-2 by full-length human ACE2. Science 367, 14441448 (2020).

29. X. Zou, K. Chen, J. Zou, P. Han, J. Hao, Z. Han, Single-cell RNA-seq data analysis on the receptor ACE2 expression reveals the potential risk of different human organs vulnerable to $2019-\mathrm{nCoV}$ infection. Frontiers of medicine, 1-8 (2020).

30. G. Goshua, A. B. Pine, M. L. Meizlish, C.-H. Chang, H. Zhang, P. Bahel, A. Baluha, N. Bar, R. D. Bona, A. J. Burns, Endotheliopathy in COVID-19associated coagulopathy: evidence from a single-centre, cross-sectional study. The Lancet Haematology, (2020).

31. F. Coperchini, L. Chiovato, L. Croce, F. Magri, M. Rotondi, The cytokine storm in COVID-19: an overview of the involvement of the chemokine/chemokine-receptor system. Cytokine \& Growth Factor Reviews, (2020).

32. C. Huang, Y. Wang, X. Li, L. Ren, J. Zhao, Y. Hu, L. Zhang, G. Fan, J. Xu, $\mathrm{X}$. Gu, Clinical features of patients infected with 2019 novel coronavirus in Wuhan, China. The lancet 395, 497-506 (2020).

33. R. E. Marques, R. Guabiraba, R. C. Russo, M. M. Teixeira, Targeting CCL5 in inflammation. Expert opinion on therapeutic targets 17, 1439-1460 (2013).

34. K. V. Sawant, K. M. Poluri, A. K. Dutta, K. M. Sepuru, A. Troshkina, R. P. Garofalo, K. Rajarathnam, Chemokine CXCL1 mediated neutrophil recruitment: Role of glycosaminoglycan interactions. Scientific reports 6, 33123 (2016).

35. L. Lasagni, M. Francalanci, F. Annunziato, E. Lazzeri, S. Giannini, L. Cosmi, C. Sagrinati, B. Mazzinghi, C. Orlando, E. Maggi, An alternatively spliced variant of CXCR3 mediates the inhibition of endothelial cell growth induced 
by IP-10, Mig, and I-TAC, and acts as functional receptor for platelet factor 4 . The Journal of experimental medicine 197, 1537-1549 (2003).

36. R. Tokunaga, W. Zhang, M. Naseem, A. Puccini, M. D. Berger, S. Soni, M. McSkane, H. Baba, H.-J. Lenz, CXCL9, CXCL10, CXCL11/CXCR3 axis for immune activation-a target for novel cancer therapy. Cancer treatment reviews 63, 40-47 (2018).

37. A. Carvalho, R. Alqusairi, A. Adams, M. Paul, N. Kothari, S. Peters, A. T. DeBenedet, SARS-CoV-2 Gastrointestinal Infection Causing Hemorrhagic Colitis: Implications for Detection and Transmission of COVID-19 Disease. The American journal of gastroenterology 115, 942-946 (2020).

38. H. J. Kim, H. Li, J. J. Collins, D. E. Ingber, Contributions of microbiome and mechanical deformation to intestinal bacterial overgrowth and inflammation in a human gut-on-a-chip. Proceedings of the National Academy of Sciences 113, E7-E15 (2016).

39. Y. Wang, D. B. Gunasekara, M. I. Reed, M. DiSalvo, S. J. Bultman, C. E. Sims, S. T. Magness, N. L. Allbritton, A microengineered collagen scaffold for generating a polarized crypt-villus architecture of human small intestinal epithelium. Biomaterials 128, 44-55 (2017). 


\section{Figures}

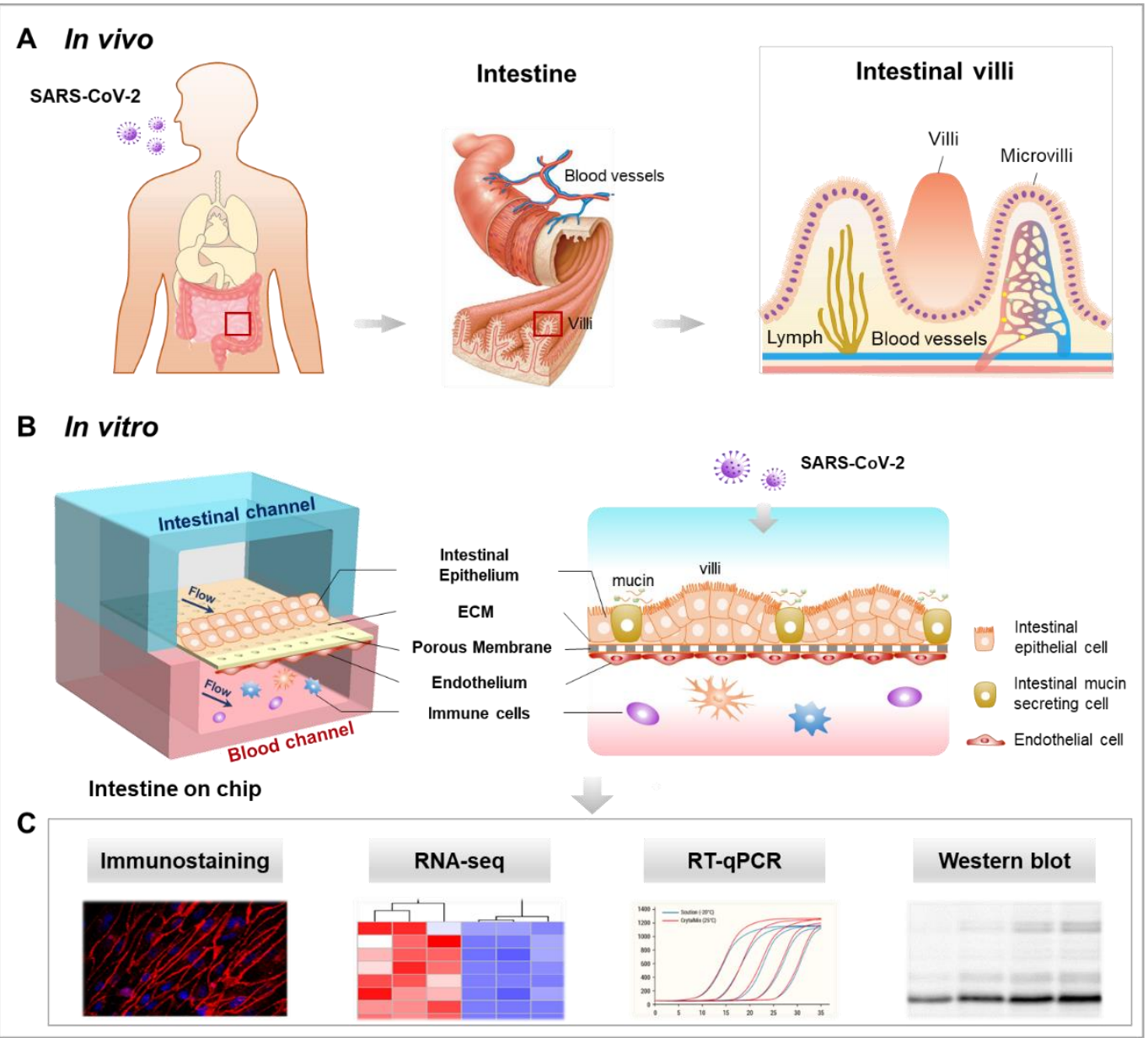

Figure 1. Schematic diagram of SARS-CoV-2-induced intestinal infection model on chip. (A) Illustration of SARS-CoV-2 infection of human small intestine in vivo. (B) The configuration SARS-CoV-2 induced intestinal infection model on chip. The device consists of upper intestinal epithelial channel (blue) and lower microvascular endothelial channel (red) separated by an ECM coated porous PDMS membrane. The intestinal barrier was established by co-culture of intestinal epithelial Caco- 2 cells and intestinal mucin secreting HT-29 cells on the top channel. Human peripheral blood mononuclear cells (PBMC) were introduced to the bottom vascular channel during the progression of virus infection. (C) The responses of the intestinal chip to SARS-CoV2 infection are analyzed using various methods. 

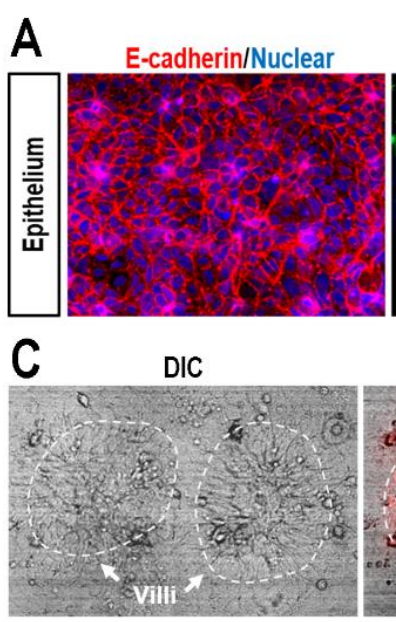

E

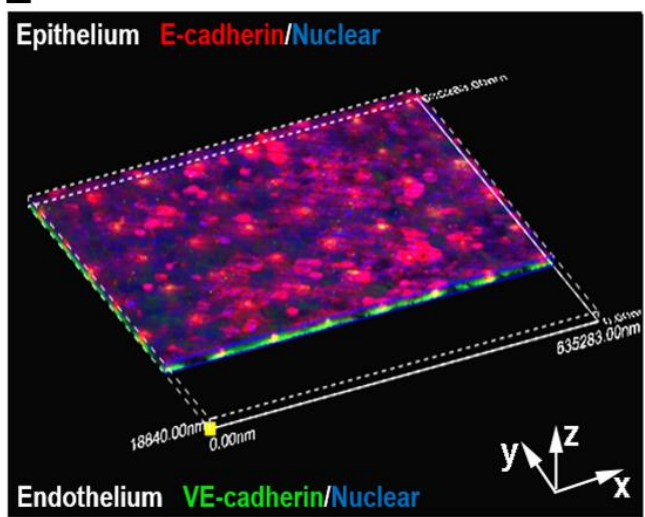

$\mathbf{F}$

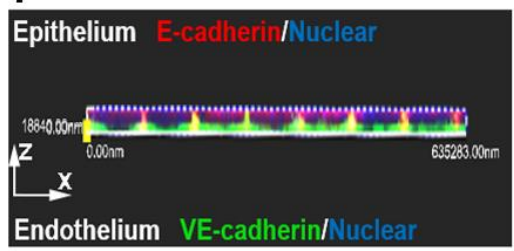

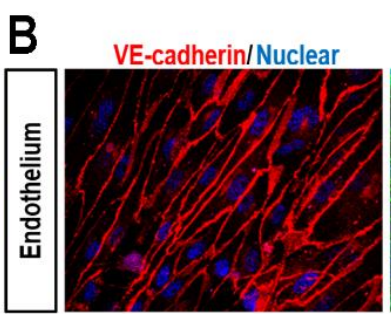

D MUC2

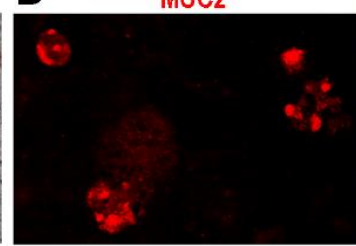

G

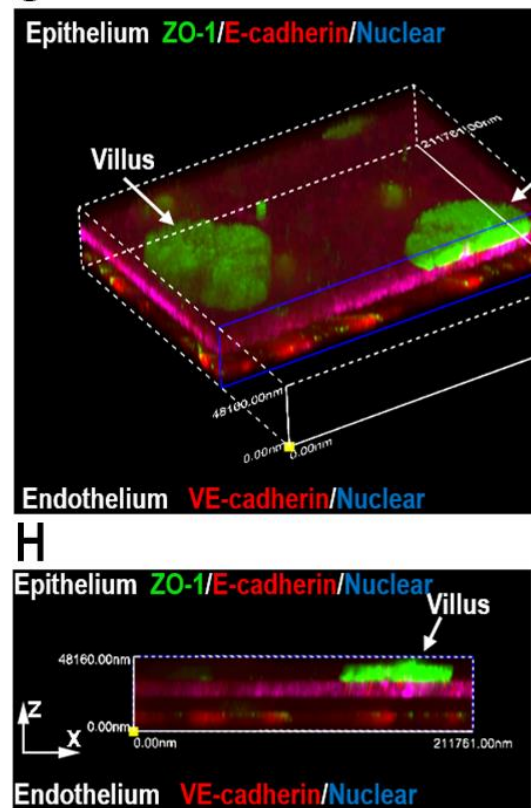

Figure 2. Characterization of intestinal epithelium and endothelium in the microengineered intestine chip. (A) Confocal micrographs showed the epithelial adhesion junctions visualized by E-cadherin and tight junctions visualized by ZO-1 in the intestine model. The intestinal villus-like structures with higher expression of ZO1 were indicated by white dotted lines. (B) Confocal micrographs showed the endothelial adhesion junctions visualized by VE-cadherin and tight junctions visualized by ZO-1 in the intestine model. (C) The DIC image showed the intestinal villus-like structure with clumps of cells (indicated by white dotted line). (D) Confocal micrographs showed scattered MUC-positive cells on endothelium. Scale bar: $50 \mu \mathrm{m}$. (E) 3D reconstructed confocal image showed intestinal epithelium (Ecadherin) and endothelium (VE-cadherin) in the intestine model. (F) Side view of intestinal epithelium (E-cadherin) and endothelium (VE-cadherin) in the intestine model. (G) 3D reconstructed confocal image showed intestinal epithelium (Ecadherin), endothelium (VE-cadherin) and intestinal villus-like structure (indicated by 
white arrow) in the microengineered intestine model. (H) Side view of intestinal epithelium (E-cadherin), endothelium (VE-cadherin) and intestinal villus-like structure (indicated by white arrow) in the microengineered intestine model. Each image represents 3 independent experiments. 

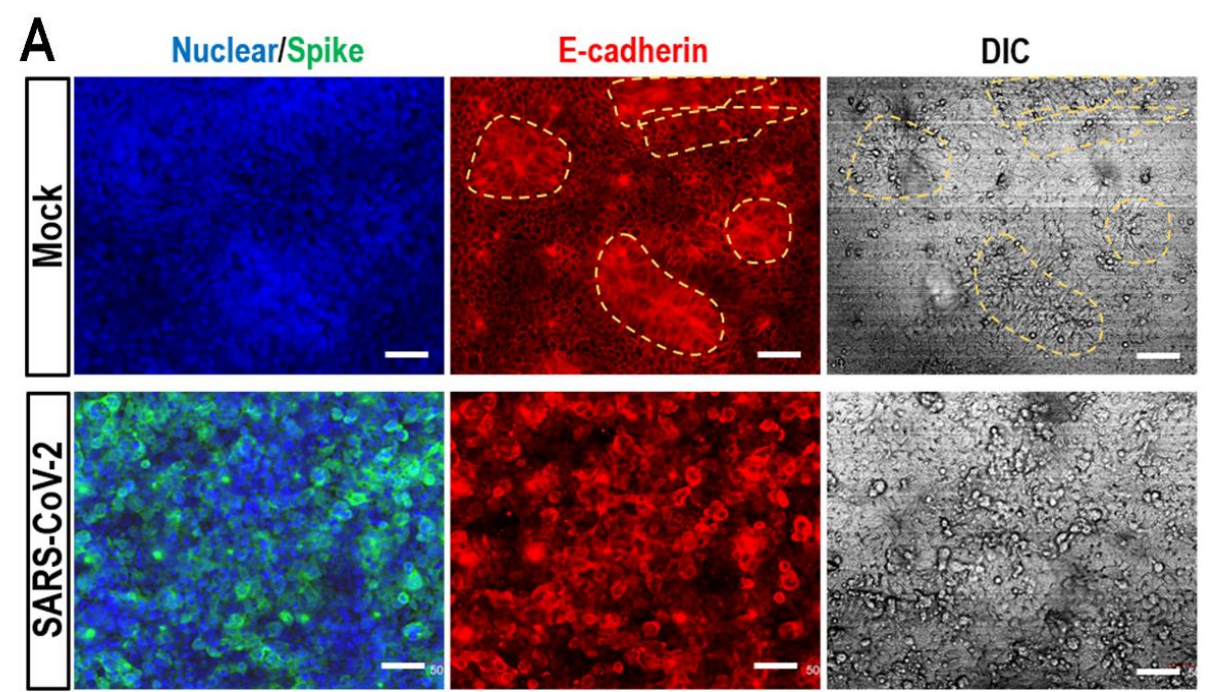

B
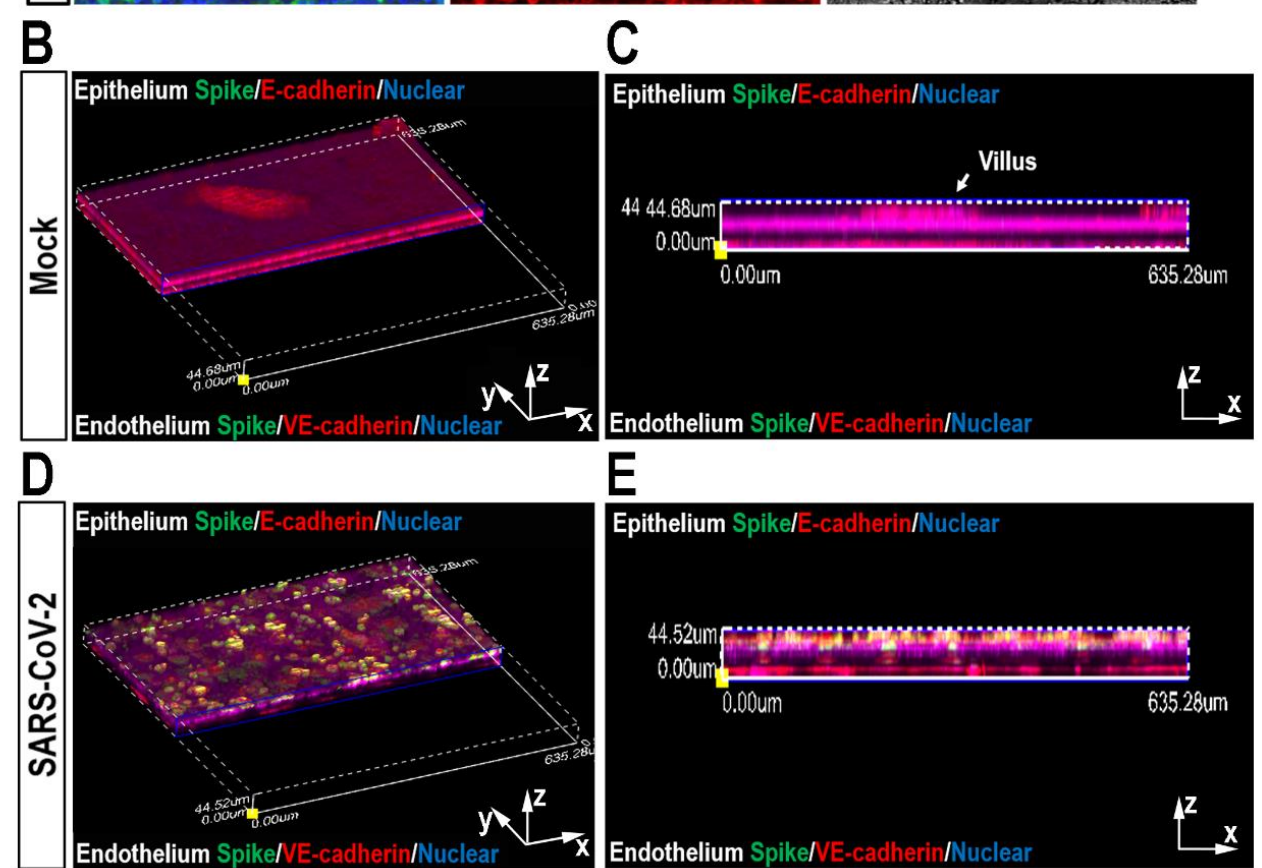

Figure 3. Examination of SARS-CoV-2 infection on the human intestine chip. (A) Confocal micrographs showed the effects of SARS-CoV-2 infection (Spike protein) on the intestinal epithelium (E-cadherin) and intestinal villus-like structure (indicated by yellow dotted line) of chip at day 3 post-infection following introduction of PBMCs into microvascular channel. (B) 3D reconstructed confocal image of mockinfected intestine model following introduction of PBMCs into microvascular channel. (C) Side view of the mock-infected intestine model. (D) 3D reconstructed confocal image of SARS-CoV-2-infected intestine model following introduction of PBMCs into microvascular channel. (E) Side view of the SARS-CoV-2-infected intestine model following introduction of PBMCs into microvascular channel. SARSCoV-2 infection was predominantly identified in epithelial layer by viral Spike protein expression. Each image represents 3 independent experiments. 


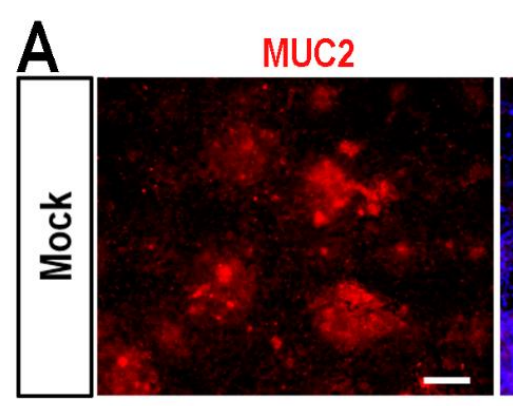

\section{Nuclear/Spike/MUC2}
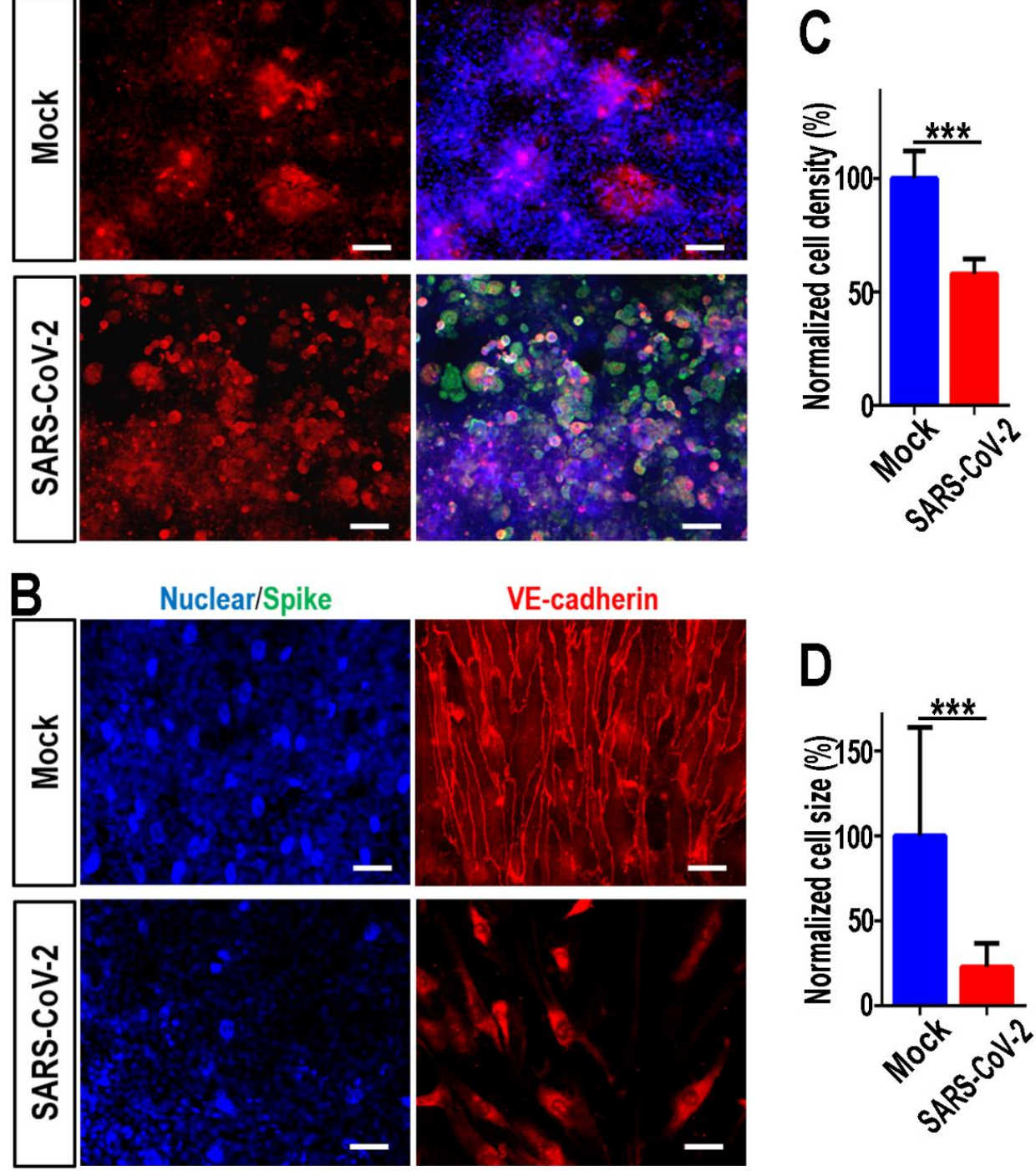

Figure 4. Morphological changes of intestinal barrier after exposure to SARS-

CoV-2. (A) Confocal micrographs showed the effects of SARS-CoV-2 infection (Spike protein) on the epithelial MUC expression in the intestine model at day 3 postinfection following introduction of PBMCs into microvascular channel. (B) Confocal micrographs showed the effects of SARS-CoV-2 infection (Spike protein) on the intestinal endothelium (E-cadherin) in microengineered intestine model following introduction of PBMCs into microvascular channel. (C, D) Quantification of endothelial cell density and size for mock- and SARS-CoV-2-infected chips. 4 chips were counted for cell density quantification in each group, and 100 cells were counted for cell size quantification in each group. Data were presented as mean \pm SD. Data were analyzed by Student's t-test $(* * *$ : $\mathrm{p}<0.001)$. 


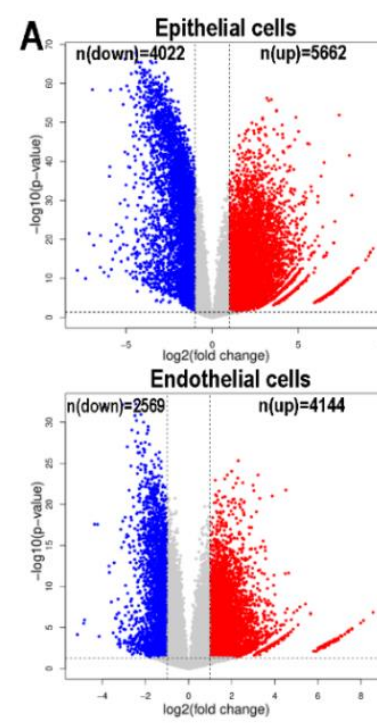

B
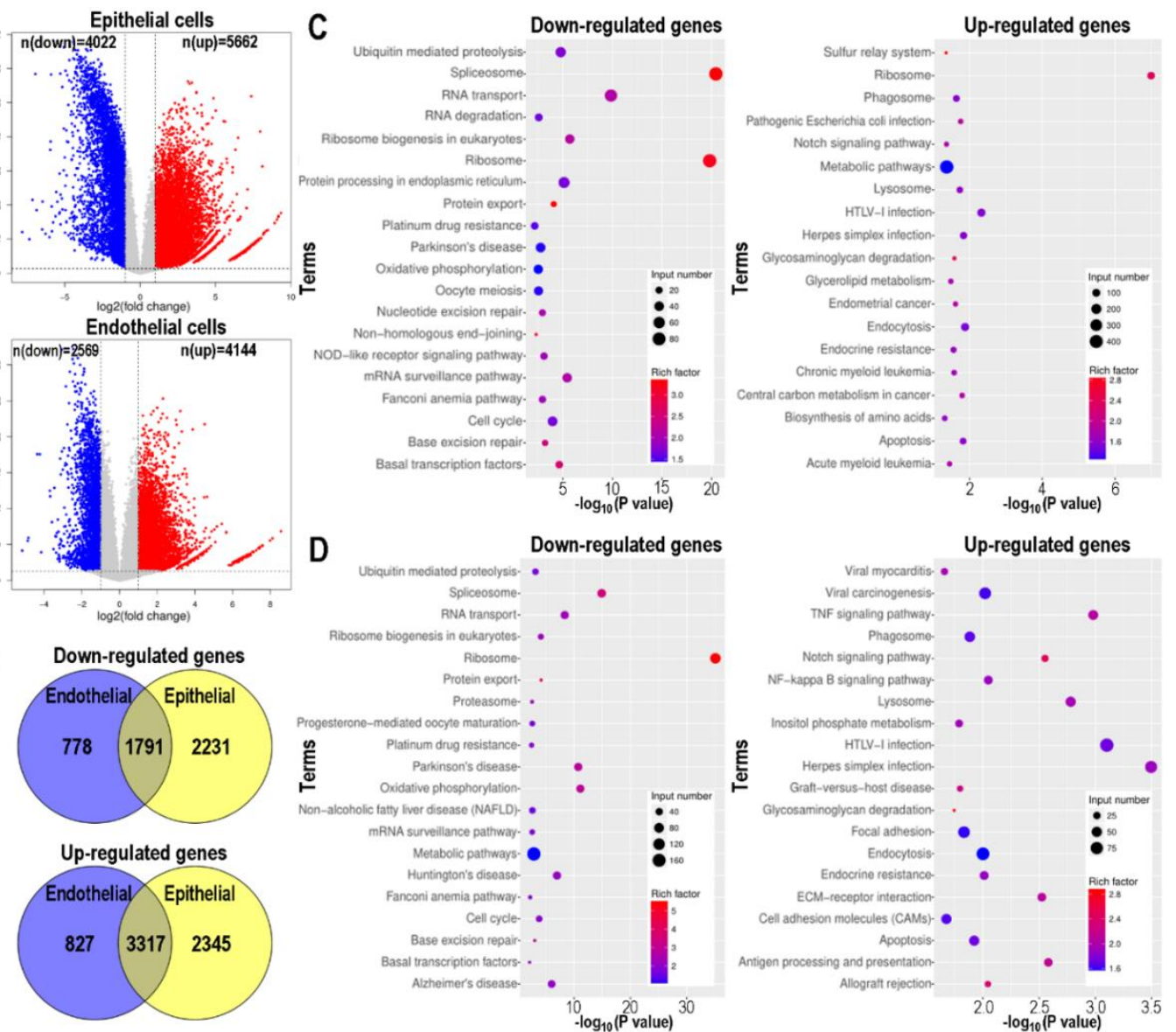

Figure 5. Transcriptional analysis of intestinal epithelial cells and endothelial cells to SARS-CoV-2 infection on chip. (A) Volcano plots showed the regulated genes of cells following SARS-CoV-2 infection. Genes differentially expressed with fold change over 2.0 and $\mathrm{p}<0.05$ were marked in color. $\mathrm{P}$ values were calculated using a two-sided, unpaired student's t-test with equal variance assumed ( $\mathrm{n}=3$ independent biological samples). (B) Venn diagrams depicting the differentially expressed genes shared or unique between each comparison. (C) KEGG pathway enrichment analysis of differentially expressed genes in intestinal epithelial cells following SARS-CoV-2 infection. (D) KEGG pathway enrichment analysis of differentially expressed genes in endothelial cells following SARS-CoV-2 infection. (E, F) The color of the dots represents the rich factor and the size represents the input number for each KEGG term. The horizontal axis indicates the significance of enrichment (- $\log 10$ (P value)), and the vertical axis indicates the enriched KEGG pathway (20 most enriched terms). 

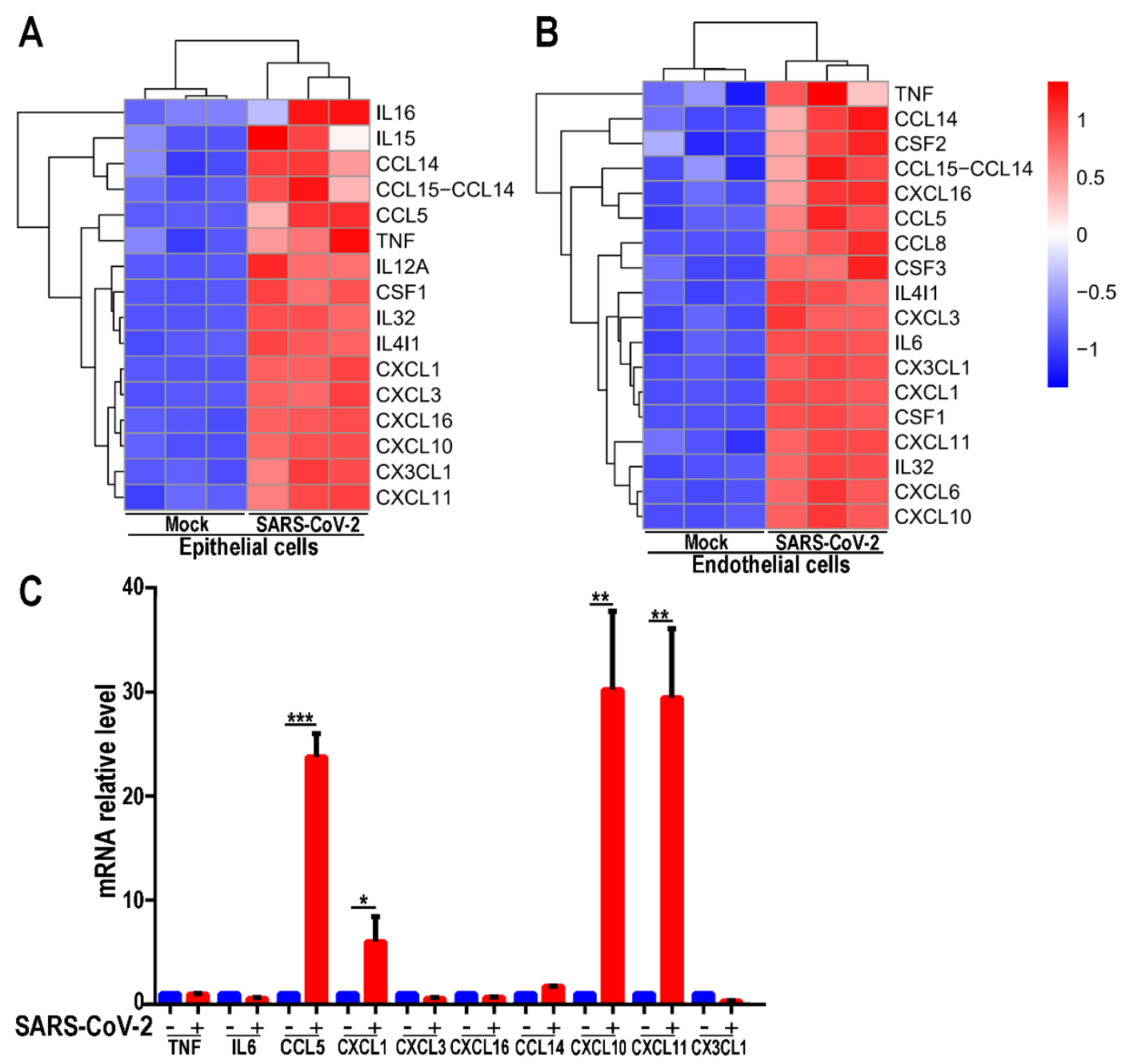

Figure 6. Assessment of immune responses in human intestine chip following SARS-CoV-2 infection (A) Heatmaps depicting the significantly up-regulated cytokine genes related in intestinal epithelial cells. (B) Heatmaps depicting the significantly up-regulated cytokine genes related in endothelial cells. Colored bars represent Z-score of $\log 2$ transformed values. (C) The relative mRNA level of indicated genes were examined by qRT-PCR. Data were presented as mean \pm SD. Data were analyzed by Student's t-test $(*$ : $p<0.05 ; * *: p<0.01 ; * * *: p<0.001) . N=3$. 